\title{
Office microlaparoscopic ovarian drilling versus conventional laparoscopic ovarian drilling for women with polycystic ovary syndrome
}

\author{
Imaduldin Salah
}

Received: 25 April 2012 / Accepted: 28 June 2012 /Published online: 19 July 2012

(C) Springer-Verlag 2012

\begin{abstract}
It is a prospective controlled study to compare the beneficial effects of office microlaparoscopic ovarian drilling (OMLOD) under augmented local anesthesia, as a new modality treatment option, compared to those following ovarian drilling with the conventional traditional $10-\mathrm{mm}$ laparoscope under general anesthesia. The study included 60 anovulatory women with polycystic ovary syndrome (PCOS) who underwent OMLOD (study group) and 60 anovulatory PCOS women, where the conventional laparoscopic ovarian drilling (LOD), using 10-mm laparoscope under general anesthesia, was performed (comparison group). Transvaginal ultrasound scan and blood sampling to measure the serum concentrations of LH, FSH, testosterone, and androstenedione had been performed before and after the procedure. Intra- and postoperative pain score in candidate women had been evaluated during the office microlaparoscopic procedure, in addition to the number of candidates who needed extra analgesia. The women undergoing OMLOD showed a good intra- and postoperative perception pain score. The number of those patients discharged within the first $2 \mathrm{~h}$ after office procedure was significantly higher, without the need for postoperative analgesia with most patients. The LH/FSH ratio and mean serum concentrations of $\mathrm{LH}$, testosterone, and free androgen index have decreased significantly after both OMLOD and LOD within a postoperative year follow-up. The mean ovarian volume decreased significantly $(P<0.05)$ within the year after both OMLOD and LOD. There were no significant differences in those results after both procedures. Intra- and postoperative augmented local anesthesia allows outpatient bilateral ovarian drilling by microlaparoscopy without general anesthesia. The relatively high pregnancy rate, the
\end{abstract}

I. Salah $(\bowtie)$

College of Medicine,

El Minya, Egypt

e-mail: imaduldin69@hotmail.com simplicity of the method, and the faster discharge time after the operation offer a new modality option for patients with PCOS, who are resistant to clomiphene citrate ovarian induction. Moreover, ovarian drilling could be performed simultaneously during the routine diagnostic microlaparoscopy and integrated in the fertility work-up investigations for those patients.

Keywords Endocrine effects · Office microlaparoscopic ovarian drilling $\cdot$ Long-term follow-up · Polycystic ovary syndrome $\cdot$ Polycystic ovaries

\section{Background}

Polycystic ovary syndrome (PCOS) is the most frequent etiological cause of anovulation observed in approximately $75 \%$ of infertile women with ovulation disorder. Patients with PCOS have various clinical symptoms and laboratory findings. PCOS presents mainly as a menstrual abnormality and infertility associated with ovulation disorder. A variety of surgical options for the treatment of PCOS have been applied during laparoscopy procedure, such as condemned laparoscopic ovarian wedge resection, electrocoagulation, and laser surgery. Laparoscopic ovarian diathermy represents an effective treatment for clomiphene citrate (CC)resistant patients and possesses numerous advantages over gonadotropin therapy, including no increased risk of ovarian hyperstimulation or multiple pregnancy and a lower incidence of spontaneous abortion $[1,2]$.

Ovarian stimulation has been shown to increase the risk of perinatal complications. Even in vitro fertilizationassisted reproductive techniques lead to a higher risk for multiple pregnancies, prematurity, low and very low birth weight, transfer to the neonatal intensive care unit, and various neonatal morbidity parameters. Thus, it has been 
proposed that ovulation induction by laparoscopic ovarian drilling (LOD) may improve the overall outcomes of pregnancies in PCOS patients. A comparison of pregnancy outcomes of women with PCOS, who had undergone LOD and non-PCOS pregnancy controls, demonstrated a significantly higher risk of hypertensive disorders and gestational diabetes mellitus for the PCOS group [3-7].

Laparoscopic ovarian drilling has been approved as the gold standard second-line treatment for anovulatory infertility due to polycystic ovary syndrome after failure to respond after course of clomiphene citrate. Not only does LOD produce high ovulation and pregnancy rates, but it also corrects the endocrine abnormalities associated with the syndrome $[4,8]$. With the development of new, smalldiameter laparoscopes that provide an optical view comparable to conventional instruments, microlaparoscopy can now be used routinely for diagnostic laparoscopy $[9,10]$. In association with pre- and postoperative local anesthesia [9-12] and new protocols for conscious sedation [4], this new approach can be used for outpatient operative microlaparoscopy without general anesthesia.

Recently, there is a great tendency to use finer laparoscopic instruments which are more suitable for office procedure under local anesthesia instead of the general anesthesia (Fig. 1). Introduction of this new technology has been limited with the narrow scope view of the finer laparoscopic instruments. A newer highly advanced microendscope, using the new fiber optic technology, has solved this great problem, offering a laparoscopic view nearly similar to the traditional $10-\mathrm{mm}$ telescope. This new updated 2-mm telescope has been recently applied for many gynecologic procedures, which were previously performed using the older traditional telescope [9-14].

In this study, a controlled prospective randomized study had been performed, recruiting 60 consenting PCOSdiagnosed patients, as a study group, to evaluate the following: (1) the feasibility of ovarian drilling during the proposed microlaparoscopy under augmented local anesthesia in terms of intraoperative pain control and postoperative analgesic requirements and (2) the efficacy of this technique compared with the conventional laparoscopic approach in another 60 PCOS-diagnosed patients as a control group, in terms of operative and discharge times and subsequent ovulation rates and pregnancy rates (PRs) within a year follow-up.

\section{Materials and methods}

Subjects

A total of 60 women with anovulatory infertility associated with PCOS underwent office microlaparoscopic ovarian drilling (OMLOD) under local anesthesia (using 2.2-mm telescope and instruments) in a private specialized fertility care center in Egypt, in addition to another 60 anovulatory women with polycystic ovaries (PCO), who underwent the conventional 10-mm laparoscopic ovarian drilling under general anesthesia. All patients included provided the data for our study. All the women had anovulatory infertility for more than 1-year duration and had been unsuccessfully treated with CC at up to $150 \mathrm{mg}$ /day for 5 days in the early follicular phase of the menstrual cycle prior to OMLOD or LOD sessions.

\section{PCOS diagnosis}

The diagnosis of PCOS in both groups of women was based on the Rotterdam criteria. (1) Evidence of hyperandrogenism, early follicular phase (defined as days $2-5$ of the menstrual cycle) serum LH/FSH ratio of $>2$, and/or raised serum androgen concentrations (testosterone $2.5 \mathrm{nmol} / \mathrm{l}$, androstenedione $10 \mathrm{nmol} / \mathrm{l}$, or free androgen index (FAI) $>4$ (Rotterdam ESHRE/ASRM-sponsored PCOS Consensus Workshop Group, Revised 2003). FAI was calculated using the formula: testosterone 100/sex hormone-binding globulin. (2) Evidence of un- or hypo-ovulation or (3) ultrasonographic evidence of PCO: ovarian stromal hypertrophy and multiple small $(6-8 \mathrm{~mm})$ follicles arranged in the periphery [9-11].

\section{OMLOD}

The microlaparoscopic system consists of a light source, a high speed pneumoperitoneum device, and a 1CCD video camera. The diameter of the scope was a malleable fiber
Fig. 1 Microlaparoscopic instruments (a) and the setup for the Office microlaparoscopy under augmented local anesthesia (b)
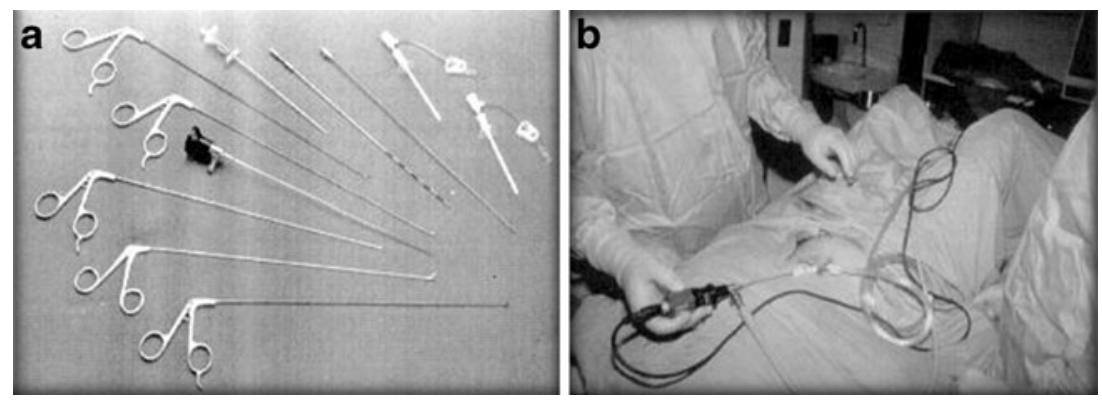
optic $2.2 \mathrm{~mm}$, and the grasping forceps, scissors, needle probes, biopsy forceps, and irrigator-aspirator were also $2.2 \mathrm{~mm}$ in diameter (all equipment made by Olympus, Tokyo, Japan). The instruments could be used by specially designed trocar (access needle; Ethicon, Cincinnati, OH, USA), which is $12-\mathrm{cm}$ long and $2.5 \mathrm{~mm}$ in diameter metal sleeve that fits over the veress needle. Premedication, consisting of $0.5 \mathrm{mg}$ of atropine sulfate and $1 \mathrm{mg} / \mathrm{kg}$ of midazolam, was given intramuscularly. Fentanyl $(1 \mathrm{mg} / \mathrm{kg})$ followed by $1.5 \mathrm{mg} / \mathrm{kg}$ of ketamine was intravenously administered through a drip infusion line.

The patient was placed in a lithotomy position. An access needle (Eticon, Tokyo, Japan) was inserted through a small incision created in the subumbilical region using the closed method, after been locally infiltrated with Xylocaine local anesthesia. Pneumoperitoneum was induced with carbon dioxide gas. Other access needles were then inserted into both sides of the hypogastric region under microlaparoscopy. Four milliliters per port of $0.25 \%$ bupivacaine were locally injected at the trocar insertion sites in advance. All the scopes and forceps used were from the microlaparoscopy set of $2.2 \mathrm{~mm}$ in outer diameter (Olympus, Tokyo, Japan). The ovarian ligament was held with holding forceps to fix it (Fig. 2).

Subsequently, the peritoneal cavity was inspected carefully, and a micrograsper was used to expose the fimbrial end of the fallopian tube at the time of chromopertubation. Chromopertubation was performed by injecting $15 \mathrm{ml}$ of methylene blue with $15 \mathrm{ml}$ of $1 \%$ mepivacaine through the uterine manipulator tubing. The ovaries were observed under the microlaparoscope. During microlaparoscopic procedure, the size of the ovary was measured using Maryland forceps. When the forceps are extended, the distance between the two arms is $1.4 \mathrm{~cm}$, and this distance was used to measure the length, width, and depth of the ovary. A high frequency 2.2-m electrocautery probe was used for ovarian drilling. The number of ovarian punctures differs according to the size and the total surface area of the ovary. A number, which ranges from 5-10 punctures, had been used for ovarian drilling. The electrocautery probe is provided with a cool water irrigation channel to rapidly cool the drilled ovary.

Fig. 2 a The microlaparoscopic image of the pelvis before the ovarian drilling procedure, which is apparently similar to the 10-mm laparoscopic view, $\mathbf{b}$ the microlaparoscopic ovarian drilling, $\mathbf{c}$ the microlaparoscopic image after the microlaparoscopic ovarian drilling procedure
At the end of the procedure, a 16-gauge, 36-cm needle was inserted through an ancillary port, and $40 \mathrm{ml}$ of $0.5 \%$ lidocaine was sprayed over the diaphragmatic vault. After the removal of all trocars, the SC tissue of the insertion sites was injected with $5 \mathrm{ml}$ of bupivacaine. Pain control during the procedure was scored on a scale of 1 to 4 , indicating excellent, good, sufficient, and poor pain control, respectively. Postoperative analgesics were given when requested by the patient. The analgesics consisted of $100 \mathrm{mg}$ of Dicolfenac Sodium (IM) or $30 \mathrm{mg}$ of ketorolac (IM). No oral postoperative medications were administered, even after hospital discharge. Patients were discharged only when completely free of discomfort.

\section{$L O D$}

Conventional laparoscopic ovarian drilling was performed using a traditional 10-mm endoscope and under general anesthesia. The operation was performed through three ports: a 10-mm laparoscope was inserted through the primary subumbilical trocar, with two additional 5-mm trocars in the lower abdomen. A grasping forceps was used for manipulation of the ovary. The unipolar needle was introduced through the other secondary site of entry. Patients underwent the same procedures as performed in the study group, such as hysteroscopy and chromopertubation, except that they received no local anesthesia. The operative time and the number of patients discharged at $2 \mathrm{~h}$ were recorded.

\section{Ultrasound scanning}

Patients underwent transvaginal scanning prior to OMLOD and LOD and after surgery. Two ultrasound machines of the same model (Toshiba, model Sonolayer SSA-250A, with a convex 6-MHz transvaginal ultrasound probe) have been used in this center during the year follow-up period. Each ovary was localized in relation to the iliac vessels and scanned from inner to outer margins in longitudinal crosssections and from upper to lower ends in transverse crosssections. The characteristic ovarian polycystic ultrasound image (increased in the ovarian stroma, the number of small follicles of more than eight in number, and the characteristic
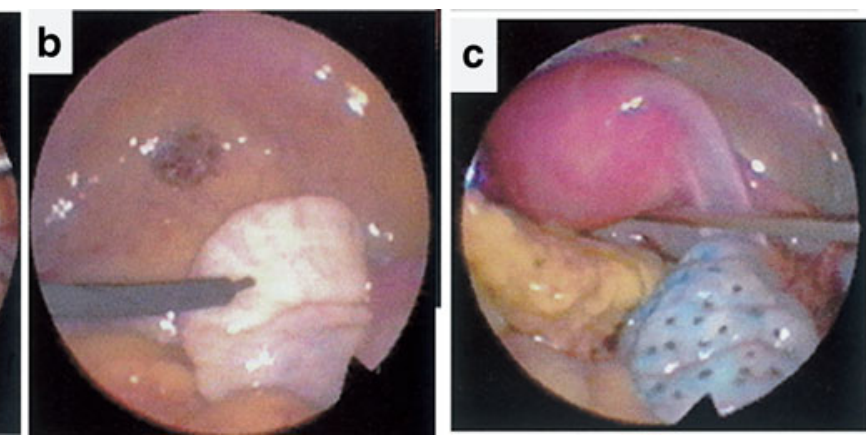

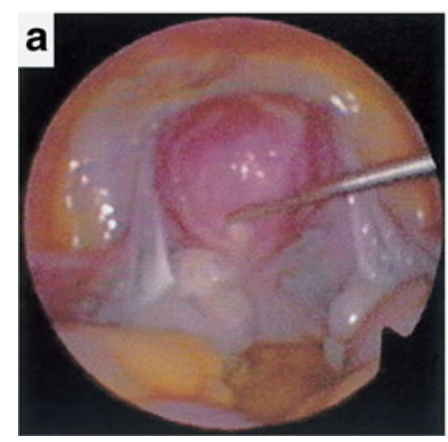


pathognomonic arrangement of those small follicles-necklace shaped) is diagnosed before the procedure and followed up postoperatively. The three diameters of the ovary were measured (longitudinal, anteroposterior, and transverse). The ovarian volume was calculated using the formula of a prolate ellipsoid: $0.523 \times$ length $\times$ width $\times$ thickness. The mean volume of the right and left ovaries was calculated for each subject.

\section{Hormonal assays}

Blood samples were taken from women in both groups early in the follicular phase (defined as days $2-5$ of the menstrual cycle - basal hormonal levels) or at a random time in women with severe oligomenorrhoea or amenorrhoea. The samples were collected from the patients prior to both procedures, and LH, FSH, LH/FSH ratio, testosterone, androstendione, and free androgen index serum hormonal levels are measured. Serum hormonal concentrations were measured using well-established assays, which have been validated in our laboratory at the Department of Clinical Chemistry, in a fertility care center laboratory. Another blood samples for hormonal assays had been taken during the year follow-up after both laparoscopic procedures, to be compared with the preoperative serum hormonal levels, performed in the same lab.

\section{Statistical analysis}

For statistical analysis, the data were entered into the Statistical Package for Social Sciences for PC version 10.0.5. Continuous data were compared by Mann-Whitney $U$ and Wilcoxon signed-ranks tests. Comparisons of categorical data were carried out using contingency table analyses. Hormonal values were compared with the postoperative values in each group with a Student's $t$ test for paired data. Ovulation, pregnancy, and miscarriage rates and the number of patients (two) discharged, $2 \mathrm{~h}$ after surgery, were evaluated by the $x$. Significance was assumed if $P<0.05$.

Findings

A total of 60 PCOS-diagnosed patients were included in this part of the study (OMLOD study group), in addition to another PCOS 60 patients (LOD comparison group). The demographic, clinical, and endocrinological characteristics of both groups of subjects had been shown in Table 1. The characteristics of the comparison subjects are also shown in the same table.

The pain score recorded for the study group was as $2.86 \pm$ 0.7 (Table 2). The operative time required for diagnostic evaluation and ovarian drilling was not significantly different between the groups. The mean discharge time and the need for additional analgesics were significantly lower in the study group than in the control group (Table 2). The proportion of patients discharged $2 \mathrm{~h}$ after surgery was significantly higher in the study group $(75.8 \%)$ than that in the comparison group (0\%) (Table 2). PRs within 1 year of follow-up were slightly higher in the study group $(65.6 \%)$ than that in the comparison group $(60 \%)$. The ovulation rate was $86.2 \%$ in the study group and $85.6 \%$ in the comparison group. The incidence of miscarriage was almost similar in both groups.

Endocrine data were reported in Table 3. LH values decreased significantly after surgery in both groups. Similarly, the serum levels showed a statistically significant reduction after surgery in both groups. In contrast, FSH levels did not change significantly after either OMLOD or the conventional laparoscopy. No complications from local anesthetics were observed during the study. The mean serum concentration of androstenedione showed no significant change shortly in both groups, but showed a significant reduction later on. The percentage of women with FAI showed a significant $(P<0.01)$ decrease from $69 \%$ prior
Table 1 The characteristics of 60 women with polycystic ovary syndrome, who had office microlaparoscopic ovarian drilling for anovulatory infertility (the study group) and 60 anovulatory PCOS women who had undergone conventional LOD (the comparison group)

\begin{tabular}{|c|c|c|c|c|}
\hline \multirow[t]{2}{*}{ Characteristic } & \multicolumn{2}{|l|}{ Cases group } & \multicolumn{2}{|c|}{ Comparison group } \\
\hline & Mean \pm SD & Range & Mean $\pm \mathrm{SD}$ & Range \\
\hline Age at follow-up (years) & $33.3 \pm 4.4$ & $21-45$ & $32.2 \pm 4.9$ & $20-44$ \\
\hline Body mass index $\left(\mathrm{kg} / \mathrm{m}^{2}\right)$ & $26.7 \pm 4.9$ & $19-4$ & $29.9 \pm 7.9$ & $18-45$ \\
\hline Duration of infertility (years) & $3.1 \pm 2.1$ & $1-12$ & $1.8 \pm 1.8$ & $1-10$ \\
\hline Serum LH (TU/1) & $14.4 \pm 7.5$ & $1.8-49.0$ & $13.0 \pm 6.2$ & $4.9-31.0$ \\
\hline Serum FSH (TU/l) & $5.4 \pm 1.5$ & $1.2-10.4$ & $5.0 \pm 1.6$ & $2.6-10.0$ \\
\hline Serum LH/FSH ratio & $2.7 \pm 1.2$ & $0.4-5.7$ & $2.6 \pm 1.4$ & $0.8-7.3$ \\
\hline Serum testosterone $(\mathrm{nmol} / \mathrm{l})$ & $2.6 \pm 1.3$ & $0.8-6.1$ & $3.2 \pm 1.2$ & $0.8-6.6$ \\
\hline Serum androstenedione (TU/1) & $9.8 \pm 4.0$ & $1.7-27$ & $11.0 \pm 3.9$ & $2.5-19.0$ \\
\hline Free androgen index & $8.6 \pm 8.2$ & $1.0-36.9$ & $11.3 \pm 7.3$ & $3.6-28.0$ \\
\hline Ovarian volume $(\mathrm{ml})$ & $11.0 \pm 3.7$ & $4.3-20.0$ & $11.5 \pm 5.2$ & $5.8-19.5$ \\
\hline
\end{tabular}


Table 2 Operative and reproductive data in patients treated by the proposed microlaparoscopy under augmented local anesthesia (study group, $n=60$ ) and after conventional 10-mm laparoscopy under general anesthesia (comparison group, $n=60$ )

\begin{tabular}{lll}
\hline Variable & Case group & Comparison group \\
\hline Intraoperative pain score & $2.8 \pm 0.7$ & - \\
Operative time (min) & $20.3 \pm 9.1$ & $28.4 \pm 7.3$ \\
Discharge time (h) & $2.4 \pm 0.7$ & $6.2 \pm 2.3$ \\
Number of patients discharged & \\
2 h after surgery (\%) & $42(71.8)$ & 0 \\
Number of patients who required & \\
Additional analgesics (\%) & $18(21.8)$ & $34(53.3)$ \\
Ovulation rate (\%) & 85.2 & 86.6 \\
PR (\%) & 65.6 & 60.0 \\
Miscarriage rate (\%) & 2.2 & 3.3 \\
\hline
\end{tabular}

to ovarian drilling to $41 \%$ shortly after surgery in both groups.

The proportion of women with ultrasound evidence of polycystic ovaries (PCO), prior to treatment and postoperatively, is shown. The results showed that the proportion of women with ultrasound evidence of PCO significantly $(P<$ $0.01)$ decreased after ovarian drilling and remained low during the 1-year follow-up periods in both groups, without a significant difference after the two procedures. The mean ovarian volume decreased significantly $(P<0.05)$ after 1 year follow-up after both LOD and OMLOD procedures.

The characteristic phenotypic picture for PCOS, including the androgenic type of obesity involving the suprapubic and waist loss, hirsutism, and acne, had been compared before and after both procedures. Those phenotypic pictures had never shown significant changes after both procedures, but with minimal improvement of both acne and the androgenic type of obesity. There was no increase in the hirsutism score; moreover, the extra hair picture had shown a slight improvement.

Other gynecologic problems had been diagnosed during both procedures. Pelvic endometriosis was confirmed in 10 women with OMLOD and 12 during LOD; all had been managed laparoscopically. Pelvic adhesions, affecting the free mobility of both tubes and ovaries, had been managed in seven women during OMLOD and in ten during LOD. Abnormally situated ovaries with disturbed tubo-ovarian relationship had been diagnosed in two cases during OMLOD and in three women during LOD. All the gynecologic problems had been laparoscopically managed without a difference between the two procedures. All those case with other problems rather than anovulation (PCOS), who had been diagnosed during both procedures, had been excluded from the study.

\section{Discussion}

The laparoscopic approach to PCOS has been described extensively [1-14]. Local anesthesia has been used for many gynecologic procedures and has been reported to reduce the postoperative pain after day-surgery diagnostic laparoscopy. Previous studies documented the implementation of microlaparoscopy without general anesthesia in terms of both diagnostic efficacy [6] and pain control during and after the procedure $[10,15-17]$. The recent development of small-diameter fiber optic microlaparoscopy allows an optical resolution comparable to that obtained with traditional 10-mm laparoscopes $[18,19]$. In addition, improvements in sedation and local anesthesia protocols [3,4] may allow microlaparoscopy to become a routine outpatient procedure.

The aim of the present study was to evaluate the feasibility and efficacy of microlaparoscopic ovarian drilling under local anesthesia and conscious sedation in terms of ovulation rates and PRs after the procedure as compared with the traditional 10-mm laparoscopic approach under general anesthesia. This technique represents a new option for gynecologists, which allows a significant reduction of androgen levels comparable to those observed after laparoscopic ovarian resection or laparoscopic electrocauterization, with the additional benefit of being a less invasive technique that can be performed as an outpatient procedure without the need for general anesthesia.
Table 3 Hormonal profile before and immediately after 1 year follow-up of ovarian drilling in patients treated by microlaparoscopy under augmented local anesthesia (study group), compared to the results after the conventional $10-\mathrm{mm}$ laparoscopic ovarian drilling under general anesthesia

\begin{tabular}{lccccc}
\hline Hormone & \multicolumn{2}{l}{ Study group } & & \multicolumn{2}{l}{ Comparison group } \\
\cline { 2 - 3 } & Before surgery & After surgery & & Before surgery & After surgery \\
\hline LH (IU/l) & $17.4 \pm 3.6$ & $14.4 \pm 2.9$ & & $18.3 \pm 3.9$ & $14.7 \pm 3.1$ \\
FSH (IU/1) & $7.1 \pm 0.7$ & $7.7 \pm 1.2$ & & $6.8 \pm 0.9$ & $7.2 \pm 1.1$ \\
LH/FSH ratio & $2.7 \pm 0.3$ & $2.1 \pm 0.1$ & & $2.7 \pm 1.2$ & $2.2 \pm 0.3$ \\
FAI (nmol/1) & $8.1 \pm 0.9$ & $6.2 \pm 0.6$ & & $8.9 \pm 1.2$ & $6.9 \pm 1.2$ \\
T (nmol/l) & $3.1 \pm 1.0$ & $2.3 \pm 0.7$ & & $3.3 \pm 0.8$ & $2.3 \pm 1.0$ \\
Androstendione (IU/l) & $9.4 \pm 1.3$ & $7.1 \pm 0.5$ & & $9.8 \pm 1.9$ & $7.6 \pm 2.3$ \\
Ovarian volume (ml) & $11.5 \pm 2.2$ & $9.9 \pm 1.3$ & & $11.7 \pm 1.7$ & $10.1 \pm 0.5$ \\
\hline
\end{tabular}


Moreover, the high PRs and ovulation rates in the followup period should encourage this approach especially in patients with PCOS, who require a diagnostic evaluation for tubal patency. The low degree of invasiveness and the modified conscious-sedation protocol of this new approach, with the use of increasing but low doses of midazolam, allow an outpatient procedure with a quick patient discharge $[3,4]$. This simple procedure appears to be particularly useful for outpatient microlaparoscopies, in which the average discharge time is 2 to $3 \mathrm{~h}$ after the operation. The effect of local anesthesia is temporary; however, this approach allows the possibility of a quicker discharge at $2 \mathrm{~h}$ after surgery [19-24].

The good intraoperative pain control reported in this study demonstrates the feasibility of operative microlaparoscopy. Even though only a small volume of $\mathrm{CO}_{2}$ is needed during the technique, the surgeon performs maneuvers to remove air from the abdomen at the end of the procedure because residual gas can accumulate under the diaphragm and cause peritoneal irritation, especially when an upright position is maintained at the time of hospital discharge. For this reason, it is useful to spray lidocaine at the end of the procedure. Moreover, reduced exposure to $\mathrm{CO}$ during 2-mm microlaparoscopy may decrease the rate of adhesion formation as compared with conventional 10-mm laparoscopy.

It had been also showed that serum testosterone concentrations decreased after both LOD and OMLOD. The proportion of women with high LH concentrations (>10 IU/l) decreased significantly from 70 to $33 \%$ shortly after OMLOD and LOD as well. There was a trend toward a decrease of serum androgen levels with the increasing postoperative duration after both OMLOD and LOD, possibly due to the effect of advancing age or due to increased loss of the cauterized ovarian stroma, the main source of the ovarian androgens. A similar trend toward a decrease of androgen levels was also observed in the comparison group, in particular, the significant reduction of serum androstenedione concentrations.

Felemban et al. [10] reported that LOD reduced both blood LH and androgens, and that, among androgens, dehydroepiandrosterone sulfate (DHEA-S) derived from the adrenal gland was significantly reduced. In this passed study, testosterone and androstenedione, probably derived from the ovary, were significantly reduced, but DHEA-S was not. Considering the fact that either OMLOD or LOD is a local procedure targeting the ovary, LOD or OMLOD is unlikely to reduce DHEA-S derived from the adrenal gland. LOD may exert their effect through a reduction of ovary-derived androgens. Amar et al. and Rossmanith et al. Also reported similar results, regarding the post drilling effects on serum androgens $[24,25]$.

The current work had shown nearly similar results to a previous research work performed in 2000, where minilaparoscope of 3.5-mm rigid lens system telescope and instruments, in contrast to the fiber optic malleable 2$\mathrm{mm}$ telescope and instruments. The limited narrow telescope view was one of the problems which faced of the minilaparoscope work [26]. The fiberoptic 2-mm telescope used in the current work offers a relatively similar image to the $10-\mathrm{mm}$ telescope. Moreover, the malleable 2-mm soft telescope and instruments have never shown any complications, with a remote possibility to injure any abdominal viscera, as they are like needle punctures, without the need for an extra repair of those needle punctures. The finer telescopic and other laparoscopic instruments had gone smoothly within the abdominal wall with the least discomfort and almost without augmented sedation, but only with local anesthetic filtration of those microlaparoscopic entries.

Ovarian drilling resulted in a significant reduction in the ovarian volume after 1-year follow-up. In one study, it had been reported on the short-term effect of ovarian drilling on the ovarian volume as measured by three-dimensional (3D) ultrasound [1-14]. They found that ovarian drilling resulted in a transient increase followed by a significant reduction in ovarian volume from a preoperative mean value of 12.2 to $6.9 \mathrm{ml} 3$ weeks after surgery. The mechanism of this reduction of the ovarian volume by ovarian drilling is not clear. In a previous study, the volume of ovarian tissue destroyed by the needle electrode in an excised ovary using a $3 \mathrm{D}$ projection of colored serial microscopic images [25-28]. They found that a single drilling would destroy an average of $0.4 \mathrm{ml}$ of stromal tissue. They concluded that $10-15$ drillings in an ovary would result in a total tissue destruction of 4-6 ml. This could account for the observed reduction of the ovarian volume.

\section{Conclusion}

In conclusion, laparoscopic ovarian drilling has been approved after extensive and prolonged research works to be the gold standard treatment tool for clomiphene citrateresistant PCOS infertile women. Technical improvements, such as smaller optical fiberoptic systems, ancillary instruments, and improved anesthetic procedures, allow these drilling procedures to be performed using microlaparoscopy under local anesthesia. OMLOD normalizes ovarian function and morphology in women with PCOS, efficiently as after conventional LOD. The question is the long-term outcome and prognosis of OMLOD, compared with those after LOD. Another question is that the patients' compliance to face the office microlaparoscopic procedure for different gynecologic and obstetric indications. The answers for those questions would appear after follow-up of the long-term outcome after OMLOD; another ongoing study and the forthcoming results might appear sooner. 
Acknowledgment I have to thank all colleagues in the university obstetrics and gynecology department for their great and kind support to this work. I am also quite grateful for Mr. Amer et al. and his colleagues for their very nice work which has added a lot to this work. I should also express my thankful feelings to all women included in the work for their patience to be followed up after the procedure and for more than 3 years. Finally, there is still an effort to be made for further implementations of this proposed microlaparoscopic procedure in another gynecologic and obstetric indications

\section{References}

1. Mercorio F, Mercorio A, Di Spiezio Sardo A, Barba GV, Pellicano M, Nappi C (2008) Evaluation of ovarian adhesion formation after laparoscopic ovarian drilling by second-look minilaparoscopy. Fertil Steril 89:1229-1233

2. Almeida OD Jr, Val-Gallas JM (1998) Office microlaparoscopy under local anesthesia in the diagnosis and treatment of chronic pelvic pain. J Am Assoc Gynecol Laparosc 5(4):407-410

3. Amer SAKS, Gopalan V, Li TC, Ledger WL, Cooke ID (2002) Long-term follow-up of patients with polycystic ovary syndrome after laparoscopic ovarian drilling: clinical outcome. Hum Reprod 17:2035-2042

4. Bayram N, vanWely M, Kaaijk EM, Bossuyt PM, van der Veen F (2004) Using an electrocautery strategy or recombinant follicle stimulating hormone to induce ovulation in polycystic ovary syndrome: randomised controlled trial. BMJ 328:192

5. Armar NA, McGarrigle HHG, Honour J, Holownia P, Jacobs HS, Lachelin GCL (1990) Laparoscopic ovarian diathermy in the management of anovulatory infertility in women with polycystic ovaries: endocrine changes and clinical outcome. Fertil Steril 53:4549

6. Malkawi HY, Qublan HS (2005) Laparoscopic ovarian drilling in the treatment of polycystic ovary syndrome: how many punctures per ovary are needed to improve the reproductive outcome? J Obstet Gynaecol Res 31:115-119

7. Risquez F, Pennehouat G, Mc Corvey R (1997) Diagnostic and operative microlaparoscopy: a preliminary multicentre report. Hum Reprod 12(8):1645-1648

8. Dahlgren E, Johansson S, Lindstedt G, Knutsson F, Oden A, Janson PO, Mattson LA, Crona N, Lundsberg PA (1992) Women with polycystic ovary syndrome wedge resected in 1956 to 1965: a long-term follow-up focusing on natural history and circulating hormones. Fertil Steril 57:505-513

9. Kaya H, Sezik M, Ozkaya O (2005) Evaluation of a new surgical approach for the treatment of clomiphene citrate-resistant infertility in polycystic ovary syndrome: laparoscopic ovarian multi-needle intervention. J Minim Invasive Gynecol 12:355-358

10. Felemban A, Tan SL, Tulandi T (2000) Laparoscopic treatment of polycystic ovaries with insulated needle cautery: a reappraisal. Fertil Steril 73:266-269

11. Barber TM, Wass JA, McCarthy MI, Franks S (2007) Metabolic characteristics of women with polycystic ovaries and oligoamenorrhoea but normal androgen levels: implications for the management of polycystic ovary syndrome. Clin Endocrinol (Oxf) 66(4):513-517

12. Gjonnaess H (1994) Ovarian electrocautery in the treatment of women with polycystic ovary syndrome (PCOS). Factors affecting the results. Acta Obstet Gynecol Scand 73:407-412
13. Gjonnaess H (1998) Late endocrine effects of ovarian electrocautery in women with polycystic ovary syndrome. Fertl Steril 69:697-701

14. Jedel E, Labrie F, Odén A et al (2011) Impact of electroacupuncture and exercise on hyperandrogenism and oligo/amenorrhea in women with polycystic ovary syndrome: a randomized controlled trial. Am J Physiol Endocrinol Metab 300:E37-45, Epub 2010 Oct 13

15. Rotterdam ESHRE/ASRM-Sponsored PCOS Consensus Workshop Group (2004) Revised 2003 consensus on diagnostic criteria and long-term health risks related to polycystic ovary syndrome (PCOS). Hum Reprod 19:41-47

16. Palomba S, Orio F Jr, Zullo F (2007) What is the best first-step therapeutic approach in treating anovulatory infertility in patients with polycystic ovary syndrome? Questions that are still unanswered. Gynecol Endocrinol 23(5):245-247

17. The Rotterdam ESHRE/ASRM-sponsored PCOS Consensus Workshop Group (2004) Revised consensus on diagnostic criteria and long-term health risks related to polycystic ovary syndrome. Fertil Steril 81:19-25

18. Keckstein G, Rossmanith W, Spatzier K, Schneider V, Borchers K, Steiner R (1990) The effect of laparoscopic treatment of polycystic ovary disease by $\mathrm{CO}_{2}$-laser or Nd:YAG laser. Surg Endosc 4:103107

19. Kovacs G, Buckler H, Bangah M, Outch K, Burger H, Healy D, Baker G, Phillips S (1991) Treatment of anovulation due to polycystic ovary syndrome by laparoscopic ovarian electrocautery. Br J Obstet Gynaecl 98:30-35

20. Li TC, Saravelos H, Chow MS, Chisabingo R, Cooke ID (1998) Factors affecting the outcome of laparoscopic ovarian drilling for polycystic ovary syndrome in women with anovulatory infertility. Br J Obstet Gynaecol 105:338-344

21. Zullo F, Pellicano M, Nappi C (2000) Minilaparoscopic ovarian drilling under local anesthesia in patients with polycystic ovary syndrome. Fertil Steril 74:376-379

22. Naether OGJ, Baukloh V, Fischer R, Kowalczyk T (1994) Longterm follow-up in 206 infertility patients with polycystic ovary syndrome after laparoscopic electrocautery of the ovarian surface. Hum Reprod 9:2342-2349

23. Takeuchi H, Jinushi M (1999) Comparison of anesthetic methods for microlaparoscopy in women with unexplained infertility. J Am Assoc Gynecol Laparosc 6:453-457

24. Seow KM, Juan CC, Hwang JL, Ho LT (2008) Laparoscopic surgery in polycystic ovary syndrome: reproductive and metabolic effects. Semin Reprod Med 26:101-110

25. Palomba S, Zullo F, Diamanti-Kandarakis E, Orio F Jr (2007) Surgery and laser diathermy. In: Diamanti-Kandarakis E, Nestler JE, Panidis D, Pasquali R (eds) Insulin resistance and polycystic ovarian syndrome. Humana, Totowa, pp 461-477

26. Fulvio Zullo MD, Massimiliano Pellicano MD, Errico Zupi MD, Maurizio Guida MD, Pasquale Mastrantonio MD, Carmine Nappi MD (2000) Minilaparoscopic ovarian drilling under local anesthesia in patients with polycystic ovary syndrome. Fertil Steril 74:376-79

27. Taskin O, Yalcinoglu AI, Kafkasli A, Burak F, Ozekici U (1996) Comparison of the effects of ovarian cauterization and gonadotropinreleasing hormone agonist and oral contraceptive therapy combination on endocrine changes in women with polycystic ovary disease. Fertil Steril 65:1115-1118

28. Tulandi T, Watkin K, Tan SL (1997) Reproductive performance and three-dimensional ultrasound volume determination of polycystic ovaries following laparoscopic ovarian drilling. Int J Infertil $42: 436-440$ 